\title{
Downregulation of AlphaB-crystallin in Retinal Pigment Epithelial Cells Exposed to Diabetes-related Stimuli In Vivo and In Vitro
}

\author{
DI WU ${ }^{1,2,3}$, SATORU KASE $^{3}$, YE LIU $^{1,2,3}$, ATSUHIRO KANDA $^{3}$, MIYUKI MURATA $^{3}$ and SUSUMU ISHIDA ${ }^{3}$ \\ ${ }^{1}$ Eye Center of the Second Affiliated Hospital of Zhejiang University, School of Medicine, Hangzhou, P.R. China; \\ ${ }^{2}$ Zhejiang Provincial Key Lab of Ophthalmology, Hangzhou, P.R. China; \\ ${ }^{3}$ Laboratory of Ocular Cell Biology and Visual Science, Department of Ophthalmology, \\ Faculty of Medicine and Graduate School of Medicine, Hokkaido University, Sapporo, Japan
}

\begin{abstract}
Background/Aim: AlphaB-crystallin plays a pivotal role in many diseases. However, the involvement of alphaB-crystallin in retinal pigment epithelial (RPE) cells with diabetes stimuli remains unknown. The aim of this study is to examine the alterations of RPE cells and alphaB-crystallin expression in diabetic models in vivo and in vitro. Materials and Methods: Diabetic conditions in mice were induced by streptozotocin (STZ). The thickness of the RPE/choroid complex was measured by optical coherence tomography $(O C T)$. Periodic acid-Schiff (PAS) staining was used to investigate the choriocapillaris in histological sections of murine eyeballs and oxidative stress was evaluated using immunofluorescence with anti-4-hydroxynonenal (HNE) antibody. AlphaB-crystallin expression was examined in the RPE/choroid complex using ELISA. Real-Time PCR was performed to evaluate the alphaB-crystallin expression in cultured human RPE cells with high glucose or following advanced glycation end-products (AGE) stimulation. Results: In diabetic mice, OCT-based RPE/choroidal layers were thickened 2 months after STZ stimulation, where PAS-positive dilated choriocapillaris was noted. Immunoreactivity of 4$H N E$ was strongly observed in the RPE layer, from which a significant number of RPE cells was lost. Meanwhile, alphaBcrystallin expression in 2-month STZ mice was significantly
\end{abstract}

This article is freely accessible online.

Correspondence to: Satoru Kase, Department of Ophthalmology, Faculty of Medicine and Graduate School of Medicine, Hokkaido University N-15, W-7, Kita-ku, Sapporo 060-8638, Japan. Tel: +81 117065944, Fax: +81 117065948, e-mail: kaseron@med.hokudai.ac.jp

Key Words: AlphaB-crystallin, retinal pigment epithelial cells, oxidative stress, high glucose, streptozotocin, advanced glycation end-products. lower compared to controls. In accordance with these results, in vitro data showed that the alphaB-crystallin expression was also significantly lower in RPE cells with high glucose or following AGE stimulation compared to untreated cells. Conclusion: In both types of diabetic models the expression of alphaB-crystallin was found to be downregulated in RPE cells and was associated with increased levels of oxidative stress.

Diabetic retinopathy (DR), a microvascular complication in patients with diabetes, is the leading cause of blindness in developed countries (1). Globally, 2.6 million people were reported to be visually impaired because of DR in 2015; however, this number is estimated to have reached 3.2 million people in 2020 (2). Disruption of the blood-retinal barrier (BRB) and the subsequent development of diabetic macular edema (DME), as well as the accumulation of leaked fluids at the macula, are the main causes of visual loss in patients with DR. The retinal pigment epithelium (RPE), a monolayer of pigmented cells lying in the interface between the photoreceptors of the neurosensory retina and the choriocapillaris, constitutes the outer BRB (3). The RPE plays an important role not only in preventing the entrance of unfavorable molecules and plasma components into the retina, but also in processing the visual cycle and protecting against photooxidation (4). Indeed, we have shown that the retina as well as the choroid play a critical role in the pathology of DR, in which RPE cells could build a biological bridge between anatomical retino-choroidal alterations $(5,6)$. Despite this, little is known about the molecular mechanisms underlying the changes in choroidal thickness found in DR.

Crystallin proteins are grouped into 3 major classes: i) alpha, ii) beta, and iii) gamma-crystallins. The two alphacrystallins, alphaA and alphaB, are the principal members of the small heat shock protein family (7). Among these, alphaB-crystallin has multiple roles, such as a chaperone of 
vascular endothelial growth factor (VEGF) or protection of RPE cells in retinal pathologies (8). Our previous study has reported that expression of alphaB-crystallin in RPE cells can play a significant role in the promotion of choroidal neovascularization with respect to age-related macular degeneration, by increasing VEGF expression as its molecular chaperone (9). Additionally, we have shown that alphaB-crystallin is expressed in neovascular endothelial cells of the proliferative DR, and colocalizes there with VEGF (8). On the other hand, alphaB-crystallin plays a pivotal role in the protection of RPE cells with oxidative stress (10). These results suggest various roles of alphaBcrystallin in certain pathological situations. The accumulation of advanced glycation end-products (AGEs), which provide a major pathogenic stimulation leading to retino-choroidal diabetic complications in human diabetic patients $(11,12)$, can downregulate alphaB-crystallin expression in vivo (13). Despite this, little is known about the alterations of alphaB-crystallin expression in RPE cells in DR. The aim of this study is to examine the alterations of RPE cells and alphaB-crystallin expression in diabetic models both in vivo and in vitro.

\section{Materials and Methods}

Animals. C57BL/6 mice aged 6 weeks were obtained from CLEA Japan (Tokyo, Japan). All animal experiments were conducted in accordance with the Association for Research in Vision and Ophthalmology Statement for the Use of Animals in Ophthalmic and Vision Research. The animal study protocol was approved by Hokkaido University (approval number: 19-0154). Procedures for murine and rat models of streptozotocin (STZ)-induced diabetes have been described in previous reports $(14,15)$. In this study, mice received an intraperitoneal injection of $80 \mathrm{mg} / \mathrm{kg}$ body weight of streptozotocin (Sigma Aldrich, St. Louis, MO, USA) at a regular h in 4 consecutive days. Mice with plasma glucose levels measuring over $250 \mathrm{mg} / \mathrm{dl} 7$ days after the last intraperitoneal injection were considered diabetic. Age-matched nondiabetic C57BL/6 mice without treatment were used as controls.

Quantification of thickness of RPE/choroid by spectral-domain optical coherence tomography (SD-OCT). For SD-OCT scans, animals were anesthetized using ketamine at $50 \mathrm{mg} / \mathrm{kg}$ body weight, and pupils were dilated with $0.5 \%$ phenylephrine and $0.5 \%$ tropicamide. Procedures for the quantification of the RPE/choroid thickness from control and STZ mice using the SD-OCT system (Spectralis, Heidelberg Engineering, Heidelberg, Germany) are described in our previous report (16).

Periodic acid-Schiff (PAS) staining. In order to identify the basement membranes and vessels in murine retino-choroidal tissues, this study employed PAS staining. Mouse eyeballs were isolated, fixed in $4 \%$ paraformaldehyde (PFA) for $1 \mathrm{~h}$ on ice, processed and embedded in paraffin. Melanin bleaching was carried out using $20 \%$ $\mathrm{H}_{2} \mathrm{O}_{2}$ for $15 \mathrm{~h}$. Sections were incubated with $0.5 \%$ periodic acid solution (ACS, Sigma Aldrich) for 2 min, then stained with Schiff's reagent (Sigma Aldrich) for $15 \mathrm{~min}$, followed by counterstaining with hematoxylin solution for $1 \mathrm{~min}$. The slides were rinsed with deionized water for $5 \mathrm{~min}$ after each step.

Immunofluorescence microscopy. For cryosections, mouse eyeballs were fixed in 4\% PFA for $1 \mathrm{~h}$ on ice, incubated in an increasing concentration of PBS/sucrose $(5,10$, and $20 \%)$, and embedded in optimal cutting temperature compound (OCT, Leica, Exton, PA, USA). Immunofluorescence analyses were performed as described previously $(17,18)$. For antibody staining, sections were washed with PBS containing $0.05 \%$ Triton $\mathrm{X}-100$ and $5 \%$ goat serum. Sections were probed with anti-4 hydroxynonenal (HNE) antibody (1:100; Abcam, Cambridge, UK). The secondary antibody was conjugated to Alexa Fluor 546 (Thermo Fisher Scientific, Waltham, MA, USA) and was used for the fluorescent detection of HNE. Nuclei were counterstained with DAPI (4',6-diamidino-2phenylindole), and sections were visualized under a Biorevo BZ9000 microscope (Keyence, Tokyo, Japan).

Enzyme-linked immunosorbent assay (ELISA). Total protein was isolated from the sonicated mouse RPE/choroid complexes using Cell lysis buffer (Cell Signaling Technology, Danvers, MA, USA). Levels of alphaB-crystallin protein in the murine RPE/choroid complexes were measured using the ImmunoSet alphaB-crystallin ELISA kit (ENZO Life Sciences, Farmingdale, NY, USA), according to the manufacturer's instructions. The concentration of alphaB-crystallin was normalized by the total protein concentration of the RPE/choroid complexes measured using bicinchoninic acid protein assay kit (Thermo Fisher Scientific).

Cell line and treatments. Human RPE (hTERT-RPE1) cells were obtained from American Type Culture Collection (Manassas, VA, USA) and cultured in DMEM (Fuji Film Wako Pure Chemicals, Osaka, Japan), supplemented with $10 \%$ fetal bovine serum (FBS; Thermo Fisher Scientific) at $37^{\circ} \mathrm{C}$ and $5 \% \mathrm{CO}_{2}$. In order to examine the alterations of alphaB-crystallin expression under high glucose concentration, hTERT-RPE1 cells were cultured using two 6-well plates. When confluent, the supernatants in all the wells were aspirated, and then DMEM containing high glucose (D-glucose with 10 to $50 \mathrm{mM}$ ) was added to wells in one plate, while DMEM containing mannitol (Wako, Osaka, Japan) was added to the wells in the other plate, as osmotic controls. AGE-bovine serum albumin (BSA) was obtained from Millipore, and was administered to RPE cells, as described in our previous report (19).

Real-time quantitative PCR ( $q P C R$ ) analysis. Total RNA was isolated using the TRI reagent (Molecular Research Center, Cincinnati, OH, USA) and reverse transcribed to cDNA using the GoScript reverse transcriptase (Promega, Madison, WI, USA). The primer sequences used for real-time qPCR were 5'- CTT TGA CCA GTT CTT CGG AG -3' (forward) and 5'- CCT CAA TCA CAT CTC CCA AC -3' (reverse) for alphaB-crystallin, and 5'- CCT GGC CAA GGT CAT CCA TG -3' (forward) and 5'- GGA AGG CCA TGC CAG TGA GC -3' (reverse) for GAPDH. Real-time qPCR was performed using the GoTaq qPCR Master Mix (Promega) and StepOne Plus Systems (Thermo Fisher Scientific). Gene expression levels were calculated using the $2^{-\mathrm{ddCT}}$ method, and all experimental samples were normalized using GAPDH as the internal control.

Statistical analyses. All the results are expressed as the mean \pm SEM (standard error of the mean). Student's $t$-test was used 

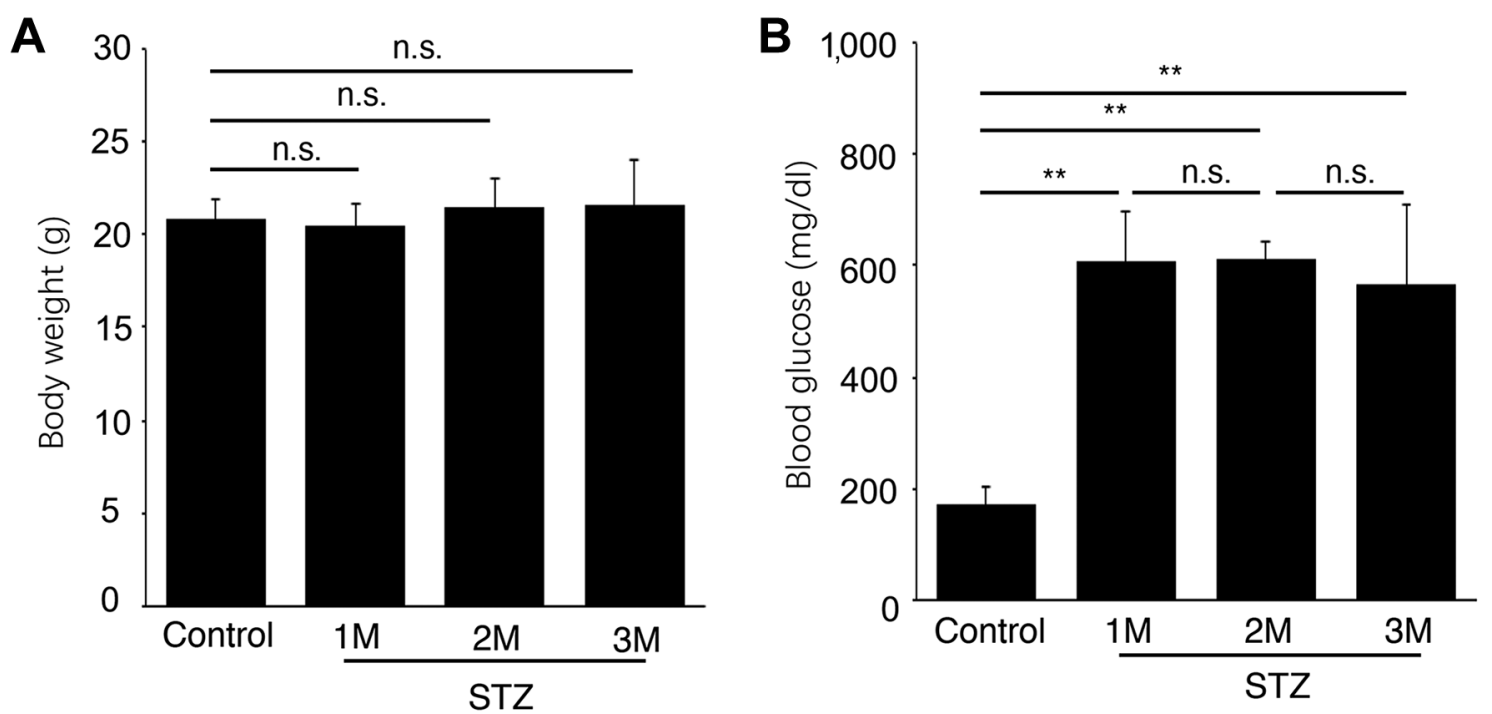

Figure 1. Systemic parameters of mice treated with streptozotocin. (A) Body weight of control mice and mice treated with streptozotocin (STZ) for 1 to 3 months $(M)(n=7-8$ each). n.s.: Not significant. (B) Blood glucose level of control mice and mice treated with STZ for 1 to 3 M. $n=7-8$ each; $* * p<0.01$.

for statistical comparison between groups, and one-way analysis of variance (ANOVA) followed by the Tukey-Kramer method as a post-hoc test was used for multiple comparison procedures. Differences between means were considered statistically significant when $p$-Values were $<0.05$.

\section{Results}

Morphological alterations of RPE/choroid with RPE cell loss in STZ-induced diabetic mice. The STZ-induced diabetic mice revealed the highest serum glucose levels and body weight at 2 months (Figure 1A and B), which, then, declined at 3 months. Observation of the RPE and choroidal layer thickness using OCT showed no significant difference at 1 month after the onset of diabetes; however, the thickness significantly increased at 2 months (Figure 2 A-D). Moreover, OCT imaging at 2 months also demonstrated that the RPE/choroid complex (seemingly a single black band) was separated into three layers, the upper of which (grey) was vague and discontinuous, the middle one whitish and the bottom one black (Figure 2C). Next, we examined whether the thickening of the RPE/choroid complexes was related to histological changes. PAS staining of the choroidal layer in STZ mice at 2 months showed irregularly larger choriocapillaris lumens compared to the control mice (Figure $3 \mathrm{~A}$ and $\mathrm{B}$ ), suggestive of the middle whitish layer in the separated RPE/choroid complex (Figure 2C). On the other hand, there was no significant difference in the choroidal medium/large vessels or stroma between STZ mice and controls, suggestive of the bottom unchanged black layer (Figure 2C). In addition, RPE cell loss was observed in diabetic mice, but not in control mice, with the number of RPE nuclei significantly lower in 2 months STZ mice compared to controls $(8.00 \pm 1.00$ and $13.33 \pm 1.53$, respectively; $p<0.01$ ) (Figure $3 \mathrm{C}$ ), pointing to the upper discontinuous grey layer (Figure 2C).

STZ-induced diabetic mice suppressed alphaB-crystallin expression and accelerated oxidative stress. Our previous study has indicated that alphaB-crystallin plays an important role in pathologies linked to murine intraocular angiogenesis (8). In this study, alphaB-crystallin protein concentration was significantly decreased in the RPE/choroid complex 2 months after STZ stimulation compared to that of control mice (Figure 4A). Next, this study focused on assessing the response to oxidative stress in the STZ-treated mice, since RPE cells are sensitive to it and commit to apoptosis in alphaB-crystallin knockout mice (10). As expected, immunofluorescence of 4HNE, an oxidative stress marker, showed an intense signal localized in the photoreceptor layer as well as in RPE and choroidal layers (Figure 4B and C). Taken together, our data suggest that STZ-induced diabetic mice reduce alphaBcrystallin protein expression and present with an increased oxidation status in the RPE/choroid complex.

AlphaB-crystallin was downregulated under high glucose stimulation in human RPE cells. To further confirm the in vivo findings of alphaB-crystallin in the RPE/choroid complex, we performed in vitro experiments using human RPE cells treated with high glucose, which is known to be an in vitro model for DR (20). Following $24 \mathrm{~h}$ treatment with 

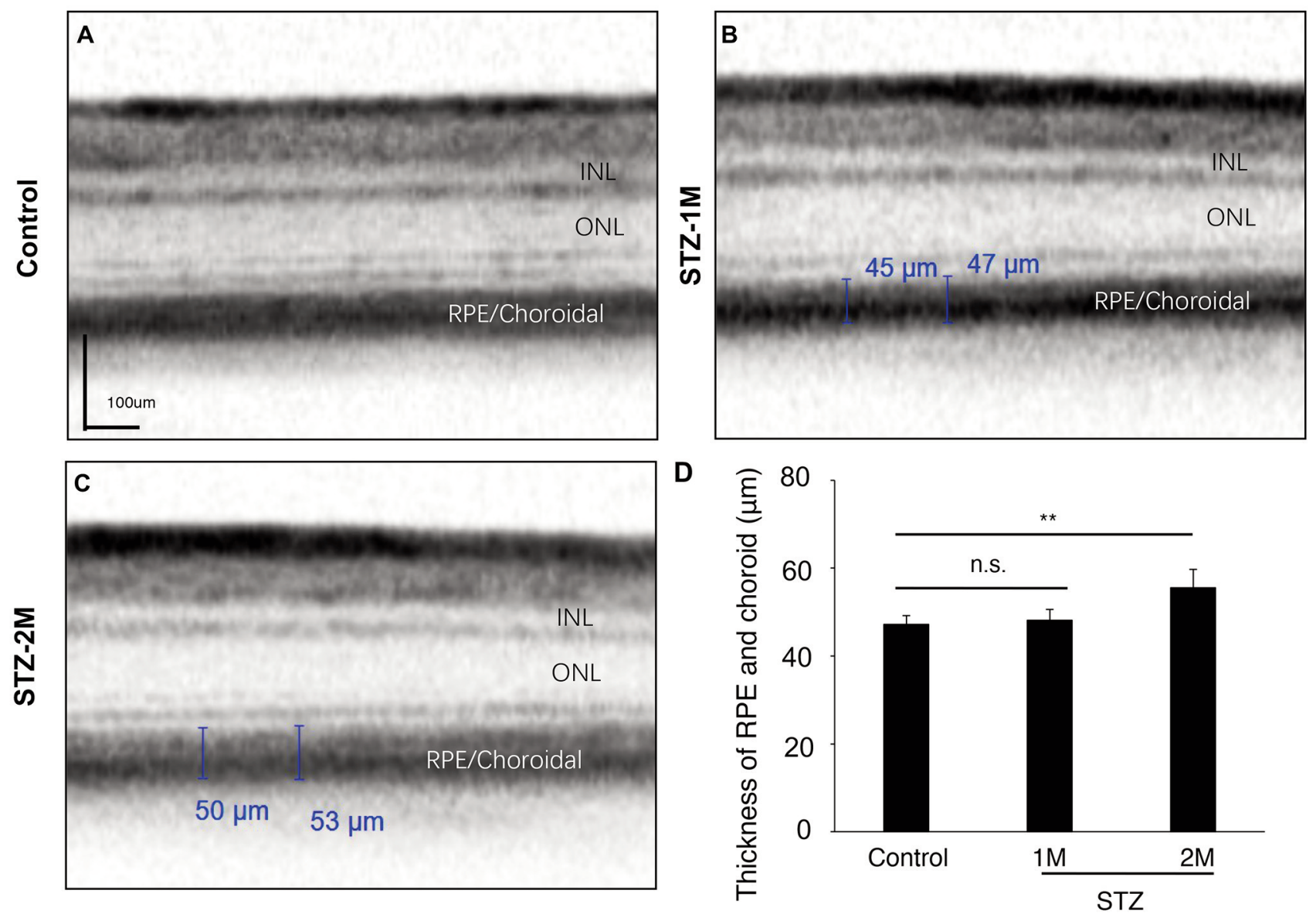

Figure 2. Retino-choroidal thickness measurements for control and diabetic mice. (A-C) Representative optical coherence tomography images of control mice and mice suffering from diabetes for 1 and 2 months. (D) Quantification of the thickness of retinal pigment epithelium (RPE) and choroidal layers in control mice and mice treated with streptozotocin (STZ) for 1 and 2 months (M). INL: Inner nuclear layer, ONL: outer nuclear layer. ${ }^{*} p<0.05,{ }^{*} p<0.01$. Scale bar $=100 \mu \mathrm{m}$.

$50 \mathrm{mM}$ glucose AlphaB-crystallin mRNA levels were significantly decreased in RPE cells in comparison to mannitol-treated control cells (Figure 5A). In contrast, alphaB-crystallin mRNA levels were unaltered during culture under $1 \%$ hypoxic conditions for up to $72 \mathrm{~h}$ (data not shown). These data indicate that it is high glucose, not hypoxia, that stimulates the reduction of alphaB-crystallin gene expression.

AlphaB-crystallin was downregulated under AGE-BSA stimuli in human cultured RPE cells. AGEs, proteins that become glycated after high glucose exposure, have been regarded as some of the main contributors for microvascular complications in diabetes $(11,21)$. Furthermore, high levels of AGEs in the vitreous fluids from patients with DR have been considered as triggers for the progression of DR (22). We previously showed strong immunoreactivity for AGEs in the RPE/choroid complex in human diabetic eyes (12). In order to investigate whether AGE contributes to alphaBcrystallin expression in RPE cells, we examined the mRNA expression levels of alphaB-crystallin with or without AGEBSA incubation. The alphaB-crystallin mRNA expression levels were significantly reduced following the addition of AGE-BSA in a dose-dependent manner (Figure 5B).

\section{Discussion}

In the present study, first, we focused on the OCT-based effect on the RPE/choroid plexus 2 months after STZ stimulation, at which time it had the greatest thickening and was accompanied by high serum glucose levels. Since histopathologically the RPE cells form a monolayer, it is likely that alterations of the RPE/choroidal thickness observed by OCT are largely caused by choroidal changes. In human diabetic eyes, we have shown that the choroidal thickness changes depending on the severity of DR (23), and 

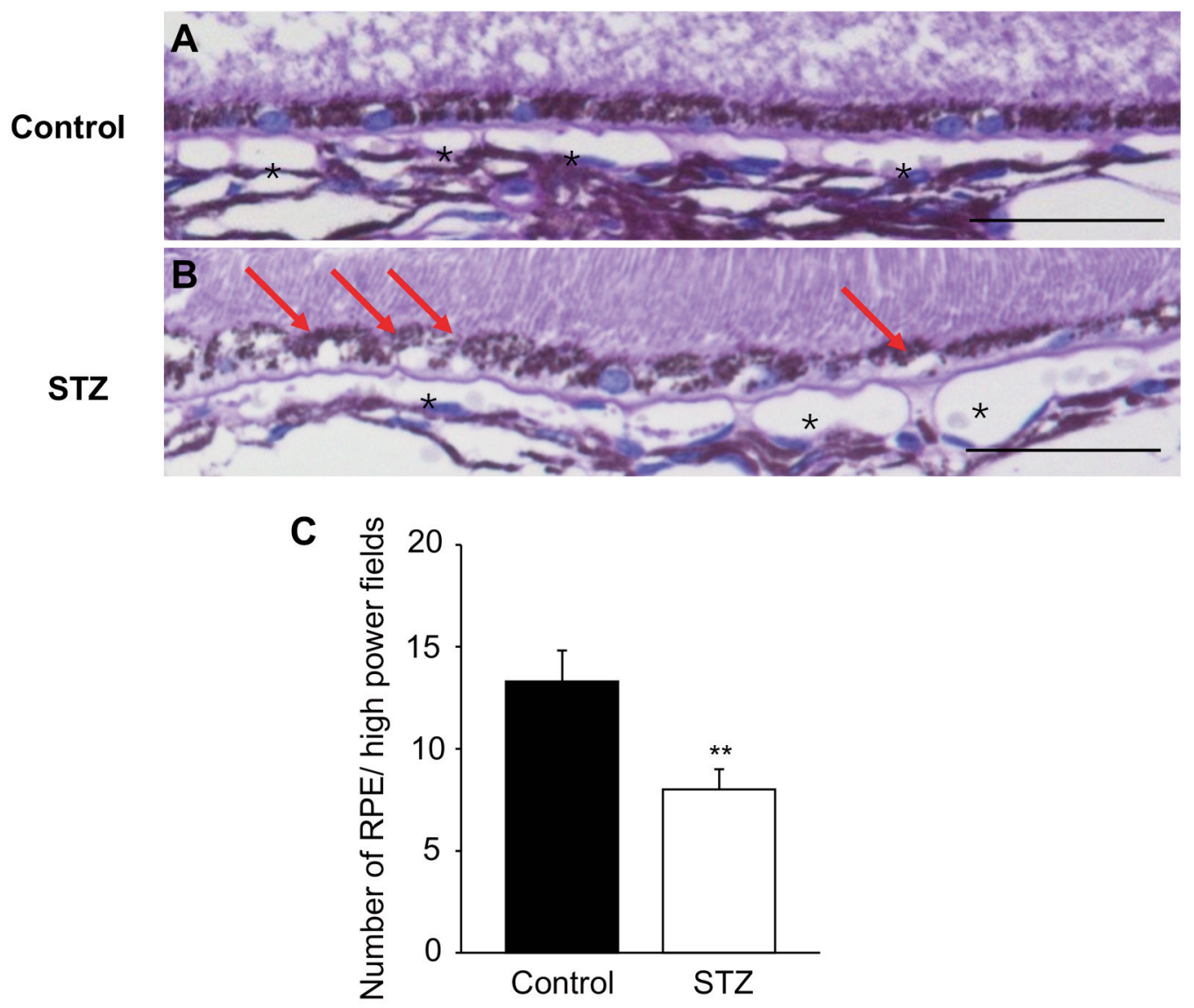

Figure 3. Morphological effects of diabetic mice on RPE and choroid layers. (A) Control mice have a normal configuration of choriocapillaris (asterisks) in the RPE monolayer. (B) Loss of RPE cell nuclei (red arrows) and dilated lumen with choriocapillaris (asterisks) are noted in 2-month diabetic mice. (C) A significantly lower number of RPE cells is detected in 2-month diabetic mice compared to controls. STZ: Streptozotocin; RPE: retinal pigment epithelium. Scale bar=50 $\mu \mathrm{m}$.

is especially thickened in patients with proliferative DR and DME $(5,24)$. It could be possible that the choroidal thickness changes observed in human diabetic eyes mimic the OCT-based RPE/choroidal thickness changes observed in STZ mice. The mechanisms underlying the changes of choroidal thickness are, however, largely unknown. In this study, we found PAS-positive abnormal dilatation of the choriocapillaris lumen together with RPE cell loss, suggesting that the vascular abnormalities might facilitate exposure of RPE cells to high glucose conditions. This study further showed that 4-HNE, an oxidative stress marker, was markedly accumulated in the RPE layer. These data suggest that the OCT-based RPE/choroidal thickening could potentially be a biomarker for vascular pathology of the choriocapillaris and for oxidative stress in RPE cells in STZtreated mice.

Next, in order to examine the possible mechanisms underlying the RPE/choroidal thickness alterations, alphaBcrystallin was tested in proteins isolated from the
RPE/choroid complex in STZ-treated mice. We found that the expression level of alphaB-crystallin was reduced in the RPE/choroid complex of STZ mice at 2 months following STZ injection. Further, we showed that the expression levels of alphaB-crystallin were also reduced in RPE cells following high glucose stimulation in vitro. Overall, alphaBcrystallin is upregulated in the retina under various pathological situations, including intraocular neovascularization (8) and in diabetic models (25), as well as in tumor cells of intraocular and periocular cancers (2628 ). The upregulated alphaB-crystallin commonly protects retinal endothelial cells from apoptosis through its phosphorylated form on serine59 and its molecular chaperone activity (9). In contrast, reduction of alphaBcrystallin causes apoptosis of RPE cells under oxidative stress (10). It has been shown that high glucose stimuli induce oxidative stress and apoptosis in cultured RPE cells (29). These results suggest that downregulation of alphaBcrystallin has led to the RPE cell damage in our DR model. 


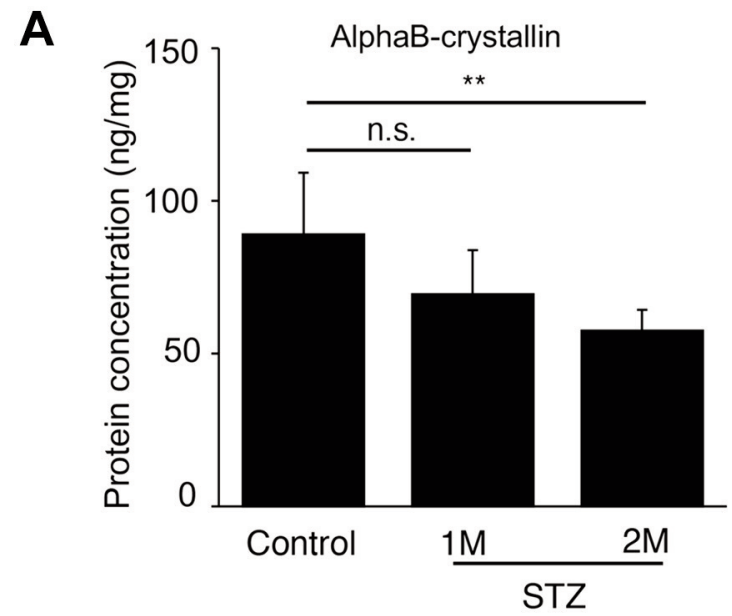

B

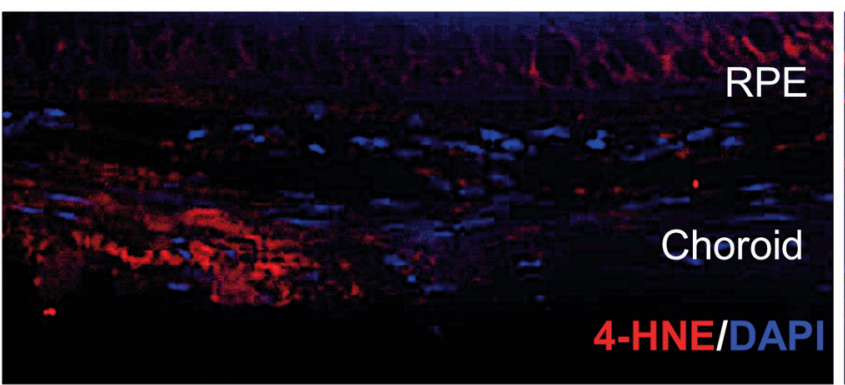

C STZ

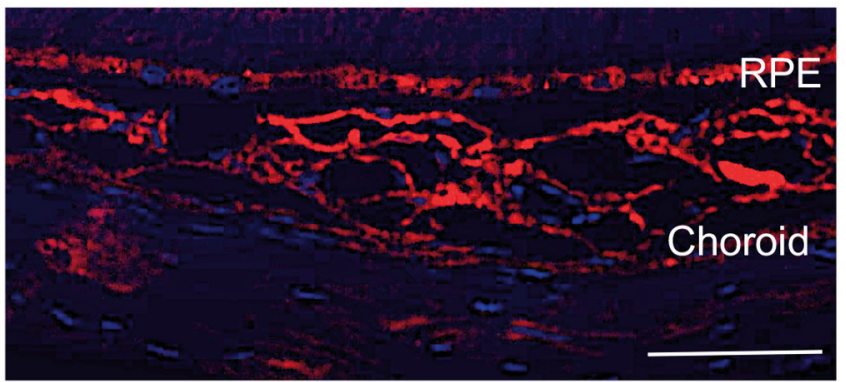

Figure 4. Impact of STZ-induced diabetic mice on alphaB-crystallin and 4-HNE in retinal pigment epithelial cells (RPE) and choroid complex. (A) Protein expression levels of alphaB-crystallin in RPE/choroid complex of control and diabetic mice. Localization of 4-HNE in the RPE/choroid complex of control $(B)$ and diabetic mice $(C)$ in red with 4',6-diamidino-2-phenylindole (DAPI) nuclear staining in blue (N=3 in each group). Scale bar $=50 \mu \mathrm{m} .{ }^{* *} p<0.05$, STZ: Streptozotocin; n.s.: Not significant.

A

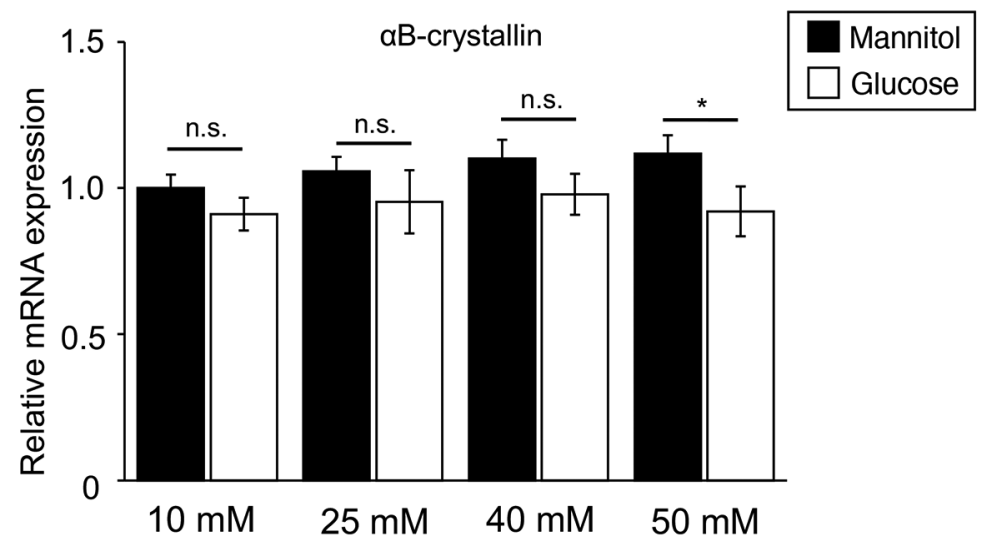

B

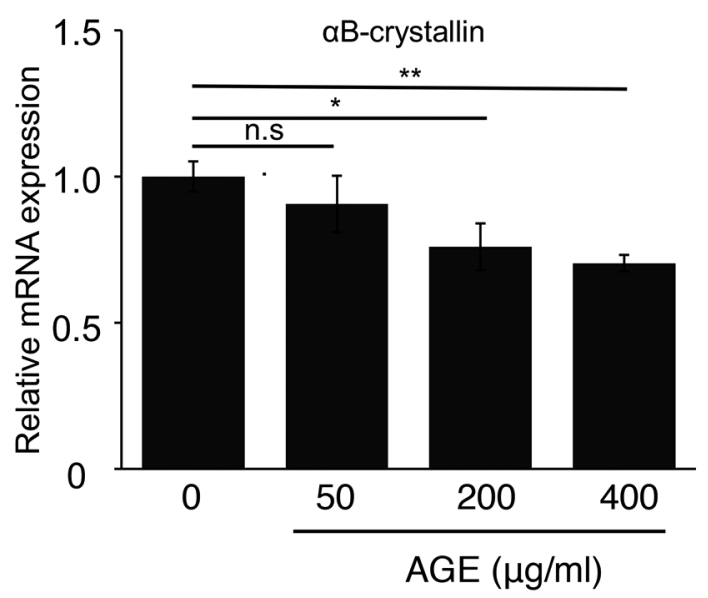

Figure 5. Gene expression levels of alphaB-crystallin in human retinal pigment epithelial (RPE) cells. (A) Real-time qPCR analysis for alphaBcrystallin in RPE cells following high glucose stimulation. (B) Real-time qPCR analysis for alphaB-crystallin in RPE cells following advanced glycation end products (AGE) stimulation ( $N=3$ in each group). $* p<0.05$, ** $p<0.01$, n.s.: Not significant. 
AGEs are major pathogenic stimuli causing various complications through their accumulation in the tissues of human diabetic patients $(11,12)$. We previously reported that injection of AGEs to the vitreous reduced alphaB-crystallin protein expression in murine retino-choroidal tissues (12). This study further demonstrated that the direct addition of AGE-BSA in cultured RPE cells downregulates alphaBcrystallin expression. These in vivo and in vitro data suggest that alphaB-crystallin could play protective roles in RPE cells under diabetic conditions. Sreekumar et al., have demonstrated that alphaB-crystallin peptides could protect RPE cells from apoptosis under mitochondrial dysfunction (30). Additional experiments assessing whether overexpression of the alphaB-crystallin gene and addition of alphaB-crystallin peptides could protect RPE cells from apoptosis would shed light into the functional role of alphaBcrystallin under diabetic conditions.

In conclusion, expression of alphaB-crystallin was downregulated in RPE cells under oxidative stress in diabetic conditions resulting in RPE cell loss. These data suggest that alphaB-crystallin may play a protective role in RPE cells under diabetic conditions.

\section{Conflicts of Interest}

The Authors declare that there are no conflicts of interest

\section{Authors' Contributions}

SK designed the research; DW, SK, YL, AK and MM performed the experiments; DW, YL and SK analyzed the data; DW, SK and SI wrote the manuscript; All Authors approved the final version.

\section{Acknowledgements}

This work was supported by Japan Agency for Medical Research and Development (AMED, project number: 18K09394) and Bayer Retina Award for unrestricted use for researches of the retina.

\section{References}

1 Lee R, Wong TY and Sabanayagam C: Epidemiology of diabetic retinopathy, diabetic macular edema and related vision loss. Eye Vis (Lond) 2: 17, 2015. PMID: 26605370. DOI: 10.1186/ s40662-015-0026-2

2 Flaxman SR, Bourne RRA, Resnikoff S, Ackland P, Braithwaite T, Cicinelli MV, Das A, Jonas JB, Keeffe J, Kempen JH, Leasher J, Limburg H, Naidoo K, Pesudovs K, Silvester A, Stevens GA, Tahhan N, Wong TY, Taylor HR and Vision Loss Expert Group of the Global Burden of Disease Study: Global causes of blindness and distance vision impairment 1990-2020: a systematic review and meta-analysis. Lancet Glob Health 5(12): e1221-e1234, 2017. PMID: 29032195. DOI: 10.1016/S2214109X(17)30393-5

3 Simó R, Villarroel M, Corraliza L, Hernández C and GarciaRamírez M: The retinal pigment epithelium: something more than a constituent of the blood-retinal barrier-implications for the pathogenesis of diabetic retinopathy. J Biomed Biotechnol 2010: 190724, 2010. PMID: 20182540. DOI: 10.1155/2010/190724

4 Strauss O: The retinal pigment epithelium in visual function. Physiol Rev 85(3): 845-881, 2005. PMID: 15987797. DOI: 10.1152/physrev.00021.2004

5 Kase S, Endo H, Takahashi M, Ito Y, Saito M, Yokoi M, Katsuta S, Sonoda S, Sakamoto T, Ishida S and Kase M: Alteration of choroidal vascular structure in diabetic macular edema. Graefes Arch Clin Exp Ophthalmol 258(5): 971-977, 2020. PMID: 32002623. DOI: $10.1007 / \mathrm{s} 00417-020-04604-\mathrm{z}$

6 Kase S, Endo H, Takahashi M, Yokoi M, Ito Y, Katsuta S, Sonoda S, Sakamoto T, Ishida S and Kase M: Involvements of choroidal vascular structures with local treatments in patients with diabetic macular edema. Eur J Ophthalmol: 11206721211027103, 2021. PMID: 34159829. DOI: 10.1177/ 11206721211027103

7 Andley UP: Crystallins in the eye: Function and pathology. Prog Retin Eye Res 26(1): 78-98, 2007. PMID: 17166758. DOI: 10.1016/j.preteyeres.2006.10.003

8 Dong Z, Kase S, Ando R, Fukuhara J, Saito W, Kanda A, Murata $\mathrm{M}$, Noda $\mathrm{K}$ and Ishida $\mathrm{S}$ : Alphab-crystallin expression in epiretinal membrane of human proliferative diabetic retinopathy. Retina 32(6): 1190-1196, 2012. PMID: 22371118. DOI: 10.1097/ IAE.0b013e318233ab9c

9 Kase S, He S, Sonoda S, Kitamura M, Spee C, Wawrousek E, Ryan SJ, Kannan R and Hinton DR: alphaB-crystallin regulation of angiogenesis by modulation of VEGF. Blood 115(16): 33983406, 2010. PMID: 20023214. DOI: 10.1182/blood-2009-01197095

10 Yaung J, Jin M, Barron E, Spee C, Wawrousek EF, Kannan R and Hinton DR: alpha-Crystallin distribution in retinal pigment epithelium and effect of gene knockouts on sensitivity to oxidative stress. Mol Vis 13: 566-577, 2007. PMID: 17438522.

11 Jakus V and Rietbrock N: Advanced glycation end-products and the progress of diabetic vascular complications. Physiol Res 53(2): 131-142, 2004. PMID: 15046548.

12 Kase S, Ishida S and Rao N: Immunolocalization of advanced glycation end products in human diabetic eyes: an immunohistochemical study. Journal of Diabetes Mellitus 01(03): 57-62, 2017. DOI: 10.4236/jdm.2011.13009

13 Kase S, Ishida S and Rao NA: Increased expression of $\alpha A-$ crystallin in human diabetic eye. Int J Mol Med 28(4): 505-511, 2011. PMID: 21617844. DOI: 10.3892/ijmm.2011.708

14 Kanda A, Noda K, Saito W and Ishida S: Aflibercept traps Galectin-1, an angiogenic factor associated with diabetic retinopathy. Sci Rep 5: 17946, 2015. PMID: 26648523. DOI: 10.1038/srep17946

15 Kim ST, Chung YY, Hwang HI, Shin HK, Choi R and Jun YH: Differential expression of BDNF and BIM in streptozotocininduced diabetic rat retina after fluoxetine injection. In Vivo 35(3): 1461-1466, 2021. PMID: 33910823. DOI: 10.21873/ invivo.12398

16 Liu Y, Noda K, Murata M, Wu D, Kanda A and Ishida S: Blockade of platelet-derived growth factor signaling inhibits choroidal neovascularization and subretinal fibrosis in mice. $\mathrm{J}$ Clin Med 9(7): 2242, 2020. PMID: 32679740. DOI: 10.3390/ jcm 9072242

17 Wu D, Kanda A, Liu Y, Kase S, Noda K and Ishida S: Galectin1 promotes choroidal neovascularization and subretinal fibrosis 
mediated via epithelial-mesenchymal transition. FASEB J 33(2): 2498-2513, 2019. PMID: 30277820. DOI: 10.1096/fj. 201801227R

18 Liu Y, Kanda A, Wu D, Ishizuka ET, Kase S, Noda K, Ichihara A and Ishida S: Suppression of choroidal neovascularization and fibrosis by a novel RNAi therapeutic agent against (Pro)renin receptor. Mol Ther Nucleic Acids 17: 113-125, 2019. PMID: 31254924. DOI: 10.1016/j.omtn.2019.05.012

19 Kanda A, Dong Y, Noda K, Saito W and Ishida S: Advanced glycation endproducts link inflammatory cues to upregulation of galectin-1 in diabetic retinopathy. Sci Rep 7(1): 16168, 2017. PMID: 29170525. DOI: 10.1038/s41598-017-16499-8

20 Huang YC, Liu SP, Chen SY, Lin JM, Lin HJ, Lei YJ, Wang YH, Huang WT, Liao WL and Tsai FJ: Increased expression of EctoNOX disulfide-thiol exchanger 1 (ENOX1) in diabetic mice retina and its involvement in diabetic retinopathy development. In Vivo 33(6): 1801-1806, 2019. PMID: 31662505. DOI: 10.21873/invivo. 11671

21 Makita Z, Radoff S, Rayfield EJ, Yang Z, Skolnik E, Delaney V, Friedman EA, Cerami A and Vlassara H: Advanced glycosylation end products in patients with diabetic nephropathy. N Engl J Med 325(12): 836-842, 1991. PMID: 1875967. DOI: 10.1056/NEJM199109193251202

22 Stitt AW, Moore JE, Sharkey JA, Murphy G, Simpson DA, Bucala R, Vlassara $\mathrm{H}$ and Archer DB: Advanced glycation end products in vitreous: Structural and functional implications for diabetic vitreopathy. Invest Ophthalmol Vis Sci 39(13): 25172523, 1998. PMID: 9856760.

23 Kase S, Endo H, Takahashi M, Saito M, Yokoi M, Ito Y, Katsuta S, Sonoda S, Sakamoto T, Ishida S and Kase M: Alteration of choroidal vascular structure in diabetic retinopathy. $\mathrm{Br} \mathrm{J}$ Ophthalmol 104(3): 417-421, 2020. PMID: 31177190. DOI: 10.1136/bjophthalmol-2019-314273

24 Kase S, Endo H, Yokoi M, Kotani M, Katsuta S, Takahashi M and Kase M: Choroidal thickness in diabetic retinopathy in relation to long-term systemic treatments for diabetes mellitus. Eur J Ophthalmol 26(2): 158-162, 2016. PMID: 26428217. DOI: $10.5301 /$ ejo. 5000676
25 Reddy VS, Raghu G, Reddy SS, Pasupulati AK, Suryanarayana $\mathrm{P}$ and Reddy GB: Response of small heat shock proteins in diabetic rat retina. Invest Ophthalmol Vis Sci 54(12): 7674-7682, 2013. PMID: 24159092. DOI: 10.1167/iovs.13-12715

26 Kase S, Parikh JG and Rao NA: Expression of heat shock protein 27 and alpha-crystallins in human retinoblastoma after chemoreduction. Br J Ophthalmol 93(4): 541-544, 2009. PMID: 18812387. DOI: $10.1136 /$ bjo.2008.145508

27 Rigas PK, Kase S and Rao NA: Expression of alpha-crystallins in human sebaceous carcinoma of the eyelid. Eur J Ophthalmol 19(5): 702-707, 2009. PMID: 19787585. DOI: 10.1177/ 112067210901900503

28 Dong Z, Kase S, Ando R, Fukuhara J, Kinoshita S, Dong Y, Takashina S, Kanda A, Noda M, Noda $K$ and Ishida S: Expression of $\alpha \mathrm{B}$-crystallin and vascular endothelial growth factor in conjunctival squamous cell carcinoma. Anticancer Res 33(9): 3745-3751, 2013. PMID: 24023305.

29 Al Sabaani N: Exendin-4 inhibits high glucose-induced oxidative stress in retinal pigment epithelial cells by modulating the expression and activation of $\mathrm{p}^{66}$ Shc. Cutan Ocul Toxicol 40(3): 175-186, 2021. PMID: 34275397. DOI: 10.1080/15569527. 2020.1844727

30 Sreekumar PG, Wang M, Spee C, Sadda SR and Kannan R: Transporter-mediated mitochondrial GSH depletion leading to mitochondrial dysfunction and rescue with $\alpha \mathrm{B}$ crystallin peptide in RPE cells. Antioxidants (Basel) 9(5): 411, 2020. PMID: 32408520. DOI: $10.3390 /$ antiox 9050411
Received August 29, 2021

Revised October 17, 2021

Accepted October 19, 2021 